\title{
Bronchoscopic thermal vapor ablation in a canine model of emphysema
}

\author{
This article was published in the following Dove Press journal: \\ International Journal of COPD \\ 25 January 2012 \\ Number of times this article has been viewed
}

\author{
Stephanie A Tuck' \\ Vanessa Lopes-Berkas² \\ Sheree Beam ${ }^{3}$ \\ Joseph C Anderson' \\ 'Uptake Medical Corp, Seattle, WA, \\ ${ }^{2}$ American Preclinical Services, Coon \\ Rapids, MN, ${ }^{3}$ Preclinical Pathology \\ Consulting Services, Ham Lake, MN, \\ USA
}

\begin{abstract}
Clinical studies indicate the potential of bronchoscopic thermal vapor ablation to result in clinically relevant improvements in severe chronic obstructive pulmonary disease patients with upper lobe-predominant emphysema. However, the mechanisms by which vapor ablation results in lung volume reduction are not fully known. This study determined the 3-month safety and efficacy of vapor ablation in a canine model of emphysema and described the histopathological changes in the lung. The cranial lobes of papain-exposed dogs were treated with a vapor dose of ten calories per gram of lung tissue $(n=8)$ or were sham treated $(n=3)$. Safety was monitored peri- and postoperatively for 3 months. Animals were then sacrificed, estimates of lung volume reduction performed, and the lungs processed for histology. Vapor ablation was associated with an average of $20 \%$ volume reduction of the treated lobes and an absence of serious adverse events. The amount of lobar volume reduction was correlated with the amount of fibrosis and atelectasis in the treated lobe. Bronchoscopic thermal vapor ablation at a dose of $10 \mathrm{cal} / \mathrm{g}$ results in lobar volume reduction associated with remodeling of the targeted tissue characterized by mature collagen formation in the absence of major adverse events.
\end{abstract}

Keywords: animal models, chronic obstructive pulmonary disease, bronchoscopy, lung volume reduction

\section{Introduction}

The NETT (National Emphysema Treatment Trial), a randomized controlled trial of medical management compared with lung volume reduction surgery (LVRS), demonstrated that LVRS in patients with emphysema, particularly those with upper lobe-predominant disease, improves lung function, exercise tolerance, and healthrelated quality of life and can improve survival in a subset of patients. ${ }^{1,2}$ However, the procedure is associated with a short-term increase in mortality and often leads to complications requiring prolonged hospitalization. Therefore, an alternative treatment to LVRS that is associated with an improved safety profile has been sought. Various minimally invasive bronchoscopic approaches are being investigated as a means to induce sustained lung volume reduction. ${ }^{3-9}$

Bronchoscopic thermal vapor ablation (BTVA) uses heated water vapor to produce lung volume reduction. The system consists of a reusable vapor generator with a disposable vapor catheter, used to deliver heated water vapor through a bronchoscope channel to targeted upper lobe emphysematous lung regions. Clinical studies indicate the potential of BTVA to induce clinically relevant improvements in severe to very severe COPD patients with upper lobe-predominant emphysema. ${ }^{10,11}$
Correspondence: Stephanie A Tuck Uptake Medical Corp, 1924 Ist Ave, Seattle, WA 98I0I, USA

$\mathrm{Tel}+\mathrm{I} 206734$ 4I0I

Fax +l 2068594557

Email stuck@uptakemedical.com 
The feasibility of using water vapor to induce lung volume reduction in an animal model was first described by Emery et al. ${ }^{12}$ They performed a dose-ranging study in dogs, which demonstrated the effectiveness of BTVA in reducing lung volume. However, the mechanisms by which BTVA treatment results in lung volume reduction were not explored. As that study was conducted in healthy dogs, the efficacy and safety of BTVA when applied to the emphysematous lung was unknown.

The objectives of the current study were two-fold: (1) to determine the 3-month safety and efficacy profile of BTVA in an animal model of emphysema, the papain-exposed dog, using a clinically relevant dose (10 cal/g), and (2) to describe the histopathological changes induced by BTVA. Part of this work was previously published in abstract form. ${ }^{13}$

\section{Methods}

All procedures were reviewed and approved by the Institutional Animal Care and Use Committee of American Preclinical Sciences (Coon Rapids, MN). Refer to Appendix 1 for additional details of methods used.

\section{Study procedures}

Eleven adult mongrel dogs (mean $\mathrm{wt}=20.9 \mathrm{~kg}$ ), previously exposed to papain by inhalation, were assigned to either a BTVA treatment $(n=8)$ or a sham treatment group $(n=3)$. Prior to treatment, measurements of vital signs and functional residual capacity (FRC) ${ }^{14}$ were taken; blood work was obtained for measurement of hematology, biochemistry, and tissue injury parameters; and computed tomography (CT) scans were taken of the chest and brain.

The BTVA procedure was then performed in anesthetized, intubated animals. A vapor catheter, connected to a vapor generator, was introduced through a bronchoscope into the airway of the lung segment selected for treatment, where an occlusion balloon was then inflated and the predetermined vapor dose (10 cal/g of lung tissue) delivered to the targeted lung segments. A total of three segments in the upper lobes were targeted for treatment: two segments in the right cranial lobe and one segment in the left cranial lobe. The order of treatment was randomized. Sham treatment consisted of placement of the catheter and balloon inflation for a time similar to that required to deliver a vapor dose of $10 \mathrm{cal} / \mathrm{g}$. Heart rate (HR), mean arterial blood pressure (MAP), and arterial oxygen saturation $\left(\mathrm{SaO}_{2}\right)$ were monitored immediately prior to and after BTVA or sham treatments. After the three vapor treatments were delivered, the animal was recovered from anesthesia and returned to housing.
Postoperative assessments consisted of blood work (postoperative Days 1, 3, 7, 30, and 90), clinical observations (daily from Day 1 to Day 10 at a minimum), FRC measurements, and chest and brain CTs at 30 and 90 days. At $\sim 90$ days posttreatment the animals were euthanized, lungs removed, and visual estimates of lobar volume reduction performed, and the lungs were processed for histological analysis.

The lung tissue slides were assessed for fibrosis, inflammation, tissue damage, and atelectasis. Fibrosis was further defined according to the maturity of the response (immature and mature granulation tissue versus mature fibrosis) as well as the distribution. Inflammation was further defined as acute, chronic with ongoing tissue damage and repair, or chronic without evidence of ongoing tissue damage and repair. The proportion of slides with a given finding, expressed as a percentage of the total number of slides examined, was reported for each lobe. The average severity of fibrosis and atelectasis for the combined treated left and right lobes was calculated for each animal and correlated with the amount of lobar volume reduction observed at necropsy.

\section{Statistical analysis}

Paired $t$-tests were used to compare pretreatment with posttreatment values, and unpaired $t$-tests were used to compare BTVA-treated and sham-treated groups. Pearson product moment correlations were used to assess the association between lobar lung volume reduction and the histological findings of fibrosis and atelectasis. A significance value of $P<0.05$ was used.

\section{Results}

The papain exposure protocol resulted in a $35 \% \pm 19 \%$ increase in FRC, measured just prior to treatment relative to FRC measured just prior to the first papain exposure.

All animals were treated with BTVA successfully without any device malfunction or perioperative adverse events. The average vapor delivery time per segment was 4.1 seconds (range 2.8-7.4 seconds). The average vapor delivery time total per animal was 12.3 seconds (range 10.7-16.4 seconds), resulting in a delivery of $479 \pm 75$ calories of energy per animal.

\section{Acute cardiovascular effects}

Pre- and posttreatment electrocardiograms were obtained in six of eight BTVA-treated dogs and three of three sham-treated dogs. All dogs maintained sinus rhythms, and no arrhythmias were observed. With the exception of 
a significant decrease in RR interval immediately after the third treatment in the BTVA dogs $(634 \mathrm{~ms}$ at pretreatment versus $565 \mathrm{~ms}$ posttreatment, $P=0.049$ ), no significant change in any other electrocardiogram interval was observed in BTVA- or sham-treated dogs (data not shown).

$\mathrm{SaO}_{2}$ was in a normal range $(>90 \%)$ at pretreatment and posttreatment for all animals with the exception of one sham-treated dog with a posttreatment $\mathrm{SaO}_{2}$ of $88 \%$. A statistically significant decrease in $\mathrm{SaO}_{2}$ was evident after the third vapor treatment in the BTVA group (mean decrease in $\mathrm{SaO}_{2}=3.5 \%, P=0.006$ ) (Figure $1 \mathrm{~A}$ ). The average decrease in $\mathrm{SaO}_{2}$ after the third treatment was similar in the shamtreated group (mean decrease in $\mathrm{SaO}_{2}=3 \%$ ), although this decrease was not statistically significant.

HR data were available for six of eight BTVA-treated dogs and three of three sham-treated dogs. No significant difference in HR between pre- and posttreatment was observed for BTVA- or sham-treated groups (Figure 1B). MAP data were available for six of eight BTVA-treated dogs and three of three sham-treated dogs. A significant increase in MAP (mean increase $=8 \mathrm{mmHg}$ ) between pre- and posttreatment was observed in the BTVA group but not the sham-treated group (Figure 1C).

\section{Clinical observations}

Clinical observations in BTVA-treated dogs were mild in nature and lasted, on average, 3 days or less (Table 1). The most frequent observations after BTVA treatment were sputum production and coughing lasting, on average, 3 days and 2 days, respectively. Other observations consisted of abnormal lung auscultation and above normal body temperature, both of which were present for an average of 1 day post-BTVA treatment. Sham-treated dogs displayed sputum production for an average of 1 day after treatment.

\section{Clinical pathology}

A transient increase in white blood cells was observed after BTVA treatment, which peaked at $17.4 \times 10^{9}$ on Day
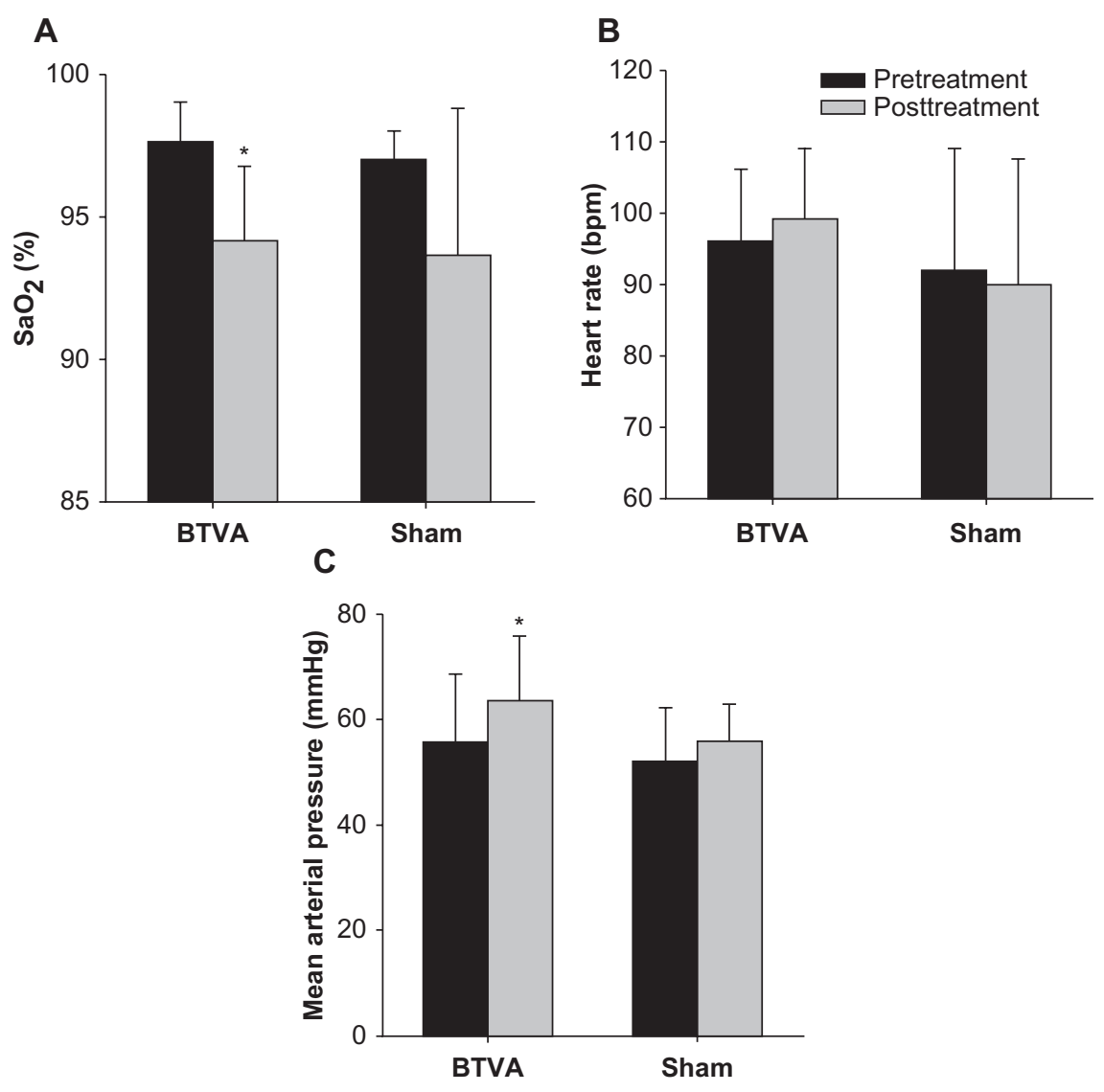

Figure I Acute cardiovascular response to bronchoscopic thermal vapor ablation (BTVA). (A) arterial oxygen saturation (SaO ${ }_{2}$ ) measured just prior to first treatment (pretreatment) and immediately after the third vapor treatment (posttreatment); $n=8$ BTVA, $n=3$ sham. (B) Heart rate measured just prior to and immediately after each treatment. Data were not analyzable in two BTVA-treated dogs. Data for all individual treatments were pooled for each animal; $n=18$ BTVA, $n=9$ sham. (C) Mean arterial pressure measured just prior to and immediately after the first and second vapor treatments. Data were not analyzable in two BTVA-treated dogs. Data for all individual treatments were pooled for each animal; $\mathrm{n}=$ II BTVA, $\mathrm{n}=8$ sham.

Note: $*$ Significantly different from pretreatment, $P<0.05$. 
Table I Clinical observations in first 10 days postbronchoscopic thermal vapor ablation (BTVA) or sham treatment

\begin{tabular}{lll}
\hline Clinical observation & \multicolumn{2}{l}{ Duration (days) } \\
\cline { 2 - 3 } & BTVA $(\mathbf{n}=\mathbf{8})$ & Sham $(\mathbf{n}=\mathbf{3})$ \\
\hline Coughing & $2 \pm 2$ & $0 \pm \mathrm{I}$ \\
Sputum & $3 \pm 2$ & $\mathrm{I} \pm \mathrm{I}$ \\
Abnormal attitude/behavior & $0 \pm \mathrm{I}$ & $0 \pm 0$ \\
Abnormal lung auscultation & $\mathrm{I} \pm \mathrm{I}$ & $0 \pm \mathrm{I}$ \\
Above normal body temperature & $\mathrm{I} \pm \mathrm{I}$ & $0 \pm 0$ \\
Abnormal arterial oxygen saturation & $0 \pm 0$ & $0 \pm 0$ \\
Death & 0 & 0 \\
\hline
\end{tabular}

Note: Data are presented as mean \pm standard deviation.

7, which would be considered slightly above normal range (Table 2). Modest elevations in generalized markers of tissue injury (creatine kinase, lactate dehydrogenase) were also observed after BTVA treatment, although the mean values posttreatment were not considered outside of the normal range for dogs. Changes in other clinical pathology parameters measured were mild and transient.

\section{CT findings}

Review of brain and chest CTs taken 30 days and 90 days posttreatment showed no evidence of stroke or pneumothorax in either BTVA- or sham-treated animals (Table 3). Evidence of consolidation in the left and right cranial lobes was apparent at 30 days and 90 days in the BTVA-treated dogs but not in the sham-treated dogs.

Volume loss in the treated cranial lobes after treatment was apparent by CT. Representative examples of images taken pre- and 3 months post-BTVA are given in Figure 2.

\section{Lobar volume reduction and gross pathology}

Lobar volume reduction was observed in $100 \%$ of the lobes treated with vapor. The average amount of visual lobar volume reduction in the treated right and left upper lobes was $20 \% \pm 8 \%$ and $19 \% \pm 15 \%$, respectively. An example of lobar volume reduction 3 months after BTVA treatment is shown in Figure 3.

\section{Functional residual capacity measurements}

FRC measurements made at 1 month and 3 months posttreatment showed no difference between the sham- and BTVA-treated groups.

\section{Histopathology}

\section{Treated cranial lobes}

A total of 805 slides from the treated right and left cranial lobes (565 slides from BTVA-treated dogs; 240 from shamtreated dogs) were assessed for fibrotic and inflammatory findings. As opposed to the cranial lobes of sham-treated dogs, where airway and parenchymal fibrosis were absent, findings of airway and parenchymal fibrosis were frequent in the cranial lobes of BTVA-treated animals. A total of 31\% of slides from the cranial lobes of BTVA-treated dogs had evidence of peribronchial fibrosis, $27 \%$ had evidence of peribronchiolar fibrosis, and $20 \%$ had evidence of parenchymal fibrosis (Table 4). The fibrosis observed in the BTVA-treated lobes was almost exclusively mature collagen; only one finding of mature granulation tissue in a BTVA-treated lobe was reported, representing $0.2 \%$ of the total slides assessed. A small proportion of slides in both treatment groups had evidence of alveolar septal fibrosis (3\% from BTVA-treated lobes; $4 \%$ from sham-treated lobes), suggesting a background level of alveolar septal fibrosis possibly related to the papain exposure.

Acute inflammation was not observed in either the BTVA-treated or sham-treated lobes at 90 days posttreatment (Table 5). A higher proportion of slides from the BTVA-treated lobes had observations of macrophages or hemosiderophages in alveolar airspaces/septa compared

Table 2 Blood markers of inflammation and tissue injury after BTVA or sham treatment

\begin{tabular}{|c|c|c|c|c|c|c|c|}
\hline Parameter & Group & Baseline & Day I & Day 3 & Day 7 & Day 30 & Day 90 \\
\hline \multirow[t]{2}{*}{ WBC $\left(\times 10^{9}\right)$} & BTVA & $5.9 \pm 1.6$ & $15.8 \pm 5.4$ & $12.7 \pm 4.6$ & $17.4 \pm 7.1$ & $7.8 \pm 3.9$ & $7.0 \pm 1.5$ \\
\hline & Sham & $6.9 \pm 1.3$ & $11.6 \pm 2.3$ & $10.3 \pm 1.7$ & $9.0 \pm 1.4$ & $6.6 \pm 0.3$ & $6.8 \pm 2.2$ \\
\hline \multirow[t]{2}{*}{ CK (U/L) } & BTVA & $146 \pm 115$ & $281 \pm 161$ & $263 \pm 328$ & $159 \pm 44$ & & \\
\hline & Sham & $190 \pm 159$ & $177 \pm 14$ & $133 \pm 74$ & $110 \pm 26$ & & \\
\hline \multirow[t]{2}{*}{ LDH (U/L) } & BTVA & $48 \pm 24$ & $153 \pm 74$ & $101 \pm 42$ & $142 \pm 57$ & & \\
\hline & Sham & $80 \pm 18$ & $111 \pm 62$ & $104 \pm 65$ & $77 \pm 49$ & & \\
\hline
\end{tabular}

Note: Values are mean \pm standard deviation.

Abbreviations: BTVA, bronchoscopic thermal vapor ablation; CK, creatine kinase; LDH, lactate dehydrogenase (not measured at Day 30 or Day 90 ); WBC, white blood cells. 
Table 3 Chest and brain CT findings at 30 and 90 days posttreatment

\begin{tabular}{|c|c|c|c|c|}
\hline \multirow[t]{3}{*}{ CT finding } & \multicolumn{4}{|c|}{ Number of animals with findings } \\
\hline & \multicolumn{2}{|c|}{ BTVA $(n=8)$} & \multicolumn{2}{|c|}{ Sham $(n=3)$} \\
\hline & 30 days & 90 days & 30 days & 90 days \\
\hline Stroke & 0 & 0 & 0 & 0 \\
\hline Pneumothorax & 0 & 0 & 0 & 0 \\
\hline Consolidation, right cranial lobe & 8 & 2 & 0 & 0 \\
\hline Consolidation, left cranial lobe & 3 & 4 & 0 & 0 \\
\hline
\end{tabular}

Abbreviations: BTVA, bronchoscopic thermal vapor ablation; CT, computed tomography.

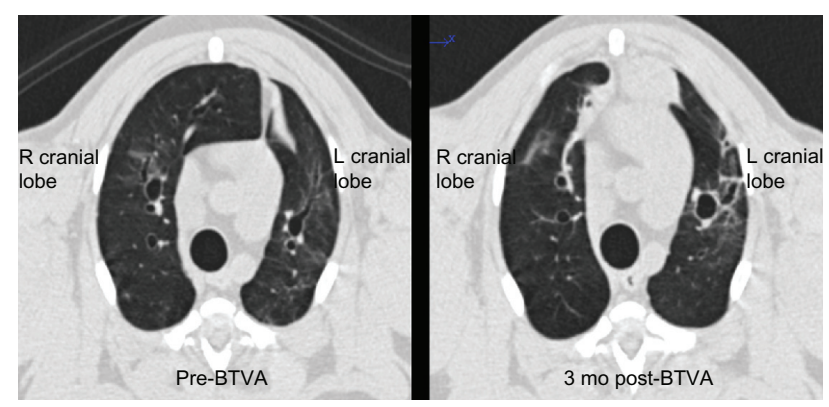

Figure 2 Axial computed tomography images of the cranial lobes before and 3 months after bronchoscopic thermal vapor ablation (BTVA) treatment.

A

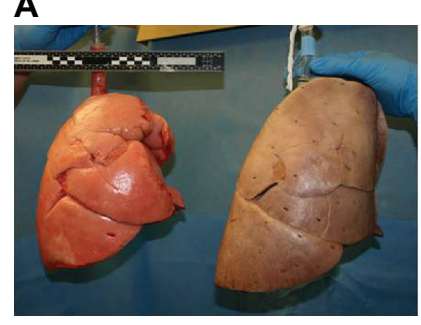

B

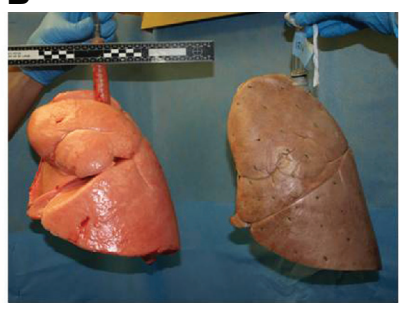

Figure 3 Example of lobar volume reduction 3 months after bronchoscopic therma vapor ablation treatment. (A) Twenty-eight percent reduction of the right cranial lobe. (B) Thirteen percent reduction of the left cranial lobe. The comparison lung on the right of each image is a dried lung from a similar-sized untreated dog.

Table 4 Fibrosis in bronchoscopic thermal vapor ablation (BTVA)- and sham-treated cranial lobes 90 days after treatment as assessed by histology

\begin{tabular}{lll}
\hline Finding & \multicolumn{2}{l}{$\begin{array}{l}\text { Percentage of slides from treated } \\
\text { cranial lobes with findings }\end{array}$} \\
\cline { 2 - 3 } & $\begin{array}{l}\text { BTVA } \\
\text { (n= 8; 565 slides) }\end{array}$ & $\begin{array}{l}\text { Sham } \\
\text { (n = 3; 240 slides) }\end{array}$ \\
& 0 & 0 \\
Immature granulation & & \\
tissue & 0.2 & 0 \\
Mature granulation & & \\
tissue & & \\
Mature collagen & 31 & 0 \\
Peribronchial & 27 & 0 \\
Peribronchiolar & 20 & 0 \\
Parenchymal & 3 & 4 \\
Alveolar septal & & \\
\hline
\end{tabular}

with sham-treated lobes (28\% versus $7 \%$ for macrophages, $18 \%$ versus $1 \%$ for hemosiderophages). However, these infiltrates were not associated with ongoing tissue injury/repair. No pleural inflammation was noted in either treatment group.

A representative example of histopathology from the cranial lobe of a BTVA-treated dog is shown in Figure 4. A linear band of mature fibrosis can be seen radiating out toward the parenchyma from a bronchus, which is also encircled by mature fibrosis. Regions of atelectasis are seen adjacent to the fibrosis.

\section{Untreated middle and caudal lobes}

Fibrosis or inflammation was infrequent in the untreated middle and lower lobes of dogs in the BTVA group. In the BTVAtreated group, single observations $(0.8 \%$ of slides reviewed) of peribronchial and parenchymal fibrosis, chronic inflammatory, and mixed inflammatory infiltrates were noted, as well as four observations (3.2\% of slides reviewed) of macrophages within airspaces (Table 6). In the untreated lobes of sham-treated dogs, the only findings were of chronic inflammatory cell infiltrates observed in three slides ( $6 \%$ of slides reviewed).

\section{Correlation between lobar volume reduction and histology findings of fibrosis and atelectasis}

Significant correlations were found between the amount of parenchymal fibrosis and atelectasis observed histologically in the treated lobes and the estimated lobar volume reduction observed grossly $(P<0.001$ and $P=0.002$ for parenchymal fibrosis and atelectasis, respectively) (Table 7). No correlation was observed between the amount of peribronchiolar or peribronchial fibrosis and lobar volume reduction.

\section{Discussion}

BTVA represents a treatment modality that can provide meaningful benefits for patients with severe emphysema. Animal studies are needed to elucidate the histopathologic understanding of the mechanism of action and the safety of BTVA. 
Table 5 Inflammation in bronchoscopic thermal vapor ablation (BTVA)- and sham-treated cranial lobes 90 days after treatment as assessed by histology

\begin{tabular}{lll}
\hline Finding & \multicolumn{2}{l}{$\begin{array}{l}\text { Percentage of slides from treated cranial lobes } \\
\text { with findings }\end{array}$} \\
\cline { 2 - 3 } & $\begin{array}{l}\text { BTVA } \\
\text { (n= 8; 565 slides) }\end{array}$ & $\begin{array}{l}\text { Sham } \\
\text { (n= 3; 240 slides) }\end{array}$ \\
\hline Acute inflammation & 0 & 0 \\
Chronic inflammation with ongoing tissue damage and repair & 0.2 & 0 \\
Chronic inflammation without ongoing tissue damage and repair & & 10 \\
$\quad$ Chronic inflammatory cell infiltrates & 6 & 3 \\
Mixed inflammatory infiltrate & 5 & 7 \\
$\quad$ Macrophages within alveolar air spaces & 28 & 1 \\
$\quad$ Alveolar septal hemosiderophages & 18 & 0 \\
Pleuritis & 0 & 0 \\
Vascular leakage, edema, hyaline membranes & 0 & \\
\hline
\end{tabular}

Note: anfiltrates around small venules in alveolar septa without necrosis or tissue destruction.

In the papain-exposed canine model of emphysema, BTVA at a vapor dose of $10 \mathrm{cal} / \mathrm{g}$ was associated with an average of $20 \%$ volume reduction of the treated lobes; no detrimental acute cardiovascular effects; no pneumothorax, respiratory distress, stroke, or death; and resolution of inflammatory processes by 3 months.

This is the first study reporting the effects of vapor ablation in an animal model of emphysema. Compared with normal lung tissue, emphysematous lungs have characteristics that may possibly affect the safety and efficacy of treatment, such as differences in perfusion, altered tissue composition, and tissue to air ratios. The distribution of emphysema in the papain-treated canine, which has been

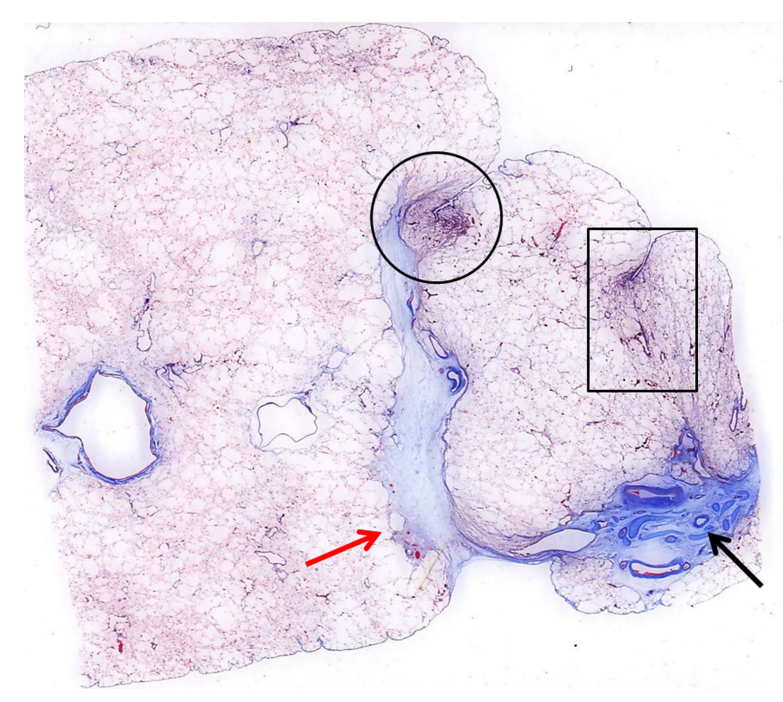

Figure 4 Histopathology example from a bronchoscopic thermal vapor ablationtreated animal.

Notes: Black arrow: collapsed bronchus encircled by mature fibrosis. Red arrow: dense band of mature collagen extending from treated bronchus to pleura. Black circle and box: areas of subpleural atelectasis at the ends of linear fibrosis. IX Masson's trichrome. described as panlobular and homogeneous, ${ }^{15,16}$ does differ from that of the target clinical population (centrilobular and upper lobe predominant), thus is a limitation of this study. However, this study does demonstrate that a therapeutically relevant BTVA dose of $10 \mathrm{cal} / \mathrm{g}$ can produce lobar volume reduction in the absence of serious adverse events in a lung with emphysema-like changes.

The vapor dose used in this study $(10 \mathrm{cal} / \mathrm{g})$ was equivalent to approximately $1.8 \mathrm{cal} / \mathrm{t}$, which was between the low $(\sim 1 \mathrm{cal} / \mathrm{mL})$ and medium $(\sim 4 \mathrm{cal} / \mathrm{mL})$ doses reported by Emery et al. ${ }^{12}$ Similar to the current study, Emery et al demonstrated that BTVA treatment induces lung volume reduction. Emery et al also described one pneumothorax-related death and possible heat-induced injury to adjacent tissues; however, those events occurred in dogs treated with medium or high $(\sim 8 \mathrm{cal} / \mathrm{mL})$ doses, which are well above the clinical doses used in humans. The current study is consistent with findings from a trial in 44 emphysematous patients who received a vapor dose of $10 \mathrm{cal} / \mathrm{g}$, noting that the treatment was well tolerated and had an acceptable safety profile. ${ }^{11}$ It should be noted that, as of yet, clinical use of BTVA has been limited to unilateral treatment only.

The regional volume reduction apparent upon visual examination of the upper lobes from the BTVA-treated canines was not reflected in FRC as measured by helium dilution. It is likely that the limitations of helium dilution in the measurement of trapped gas as well as the papain-induced model (predominantly homogeneous emphysema) explain the discrepancy between the clear visual reduction in lobar volume and the measurement of FRC.

The mechanism of action for lung volume reduction after BTVA treatment has not been fully understood. It is expected that the initial thermal response to the treated airways and 
Table 6 Histopathology findings in untreated middle and caudal lobes 90 days after treatment

\begin{tabular}{lll}
\hline Finding & $\begin{array}{l}\text { Percentage of slides from untreated middle } \\
\text { and caudal lobes with findings }\end{array}$ & $\begin{array}{l}\text { Sham } \\
\text { (n= 3; 50 slides) }\end{array}$ \\
\cline { 2 - 3 } & $\begin{array}{l}\text { BTVA } \\
\text { (n= 8; I 25 slides) }\end{array}$ & 0 \\
\hline Granulation tissue & 0 & 0 \\
Mature collagen & & 0 \\
$\quad$ Peribronchial & 0.8 & 0 \\
Peribronchiolar & 0 & 0 \\
Parenchymal & 0.8 & 0 \\
$\quad$ Alveolar septal & 0 & 0 \\
Acute inflammation & 0 & 0.0 \\
Chronic inflammation & 0 & 0 \\
Chronic inflammation without ongoing tissue damage and repair & & 0 \\
$\quad$ Chronic inflammatory cell infiltrates & 0.8 & 0 \\
$\quad$ Mixed inflammatory infiltrate & 0.8 & 0 \\
Macrophages within alveolar air spaces & 3.2 & 0 \\
$\quad$ Alveolar septal hemosiderophages & 0 & 0 \\
Pleuritis & 0 & 0 \\
Vascular leakage, edema, hyaline membranes & 0 & 0 \\
\hline
\end{tabular}

Abbreviation: BTVA, bronchoscopic thermal vapor ablation.

parenchyma results in a localized inflammatory response. This initial inflammatory response is followed by a healing process, which leads to permanent remodeling of the lung tissue. Although this study does not address early inflammatory events, the histology findings 3 months after treatment establish that fibrosis (in particular, parenchymal fibrosis) and distal atelectasis in the treated regions are important effects of vapor ablation. Scar tissue formed during the remodeling process replaces diseased, hyperinflated lung parenchyma. Because the scar tissue occupies a much smaller volume from which it derives, the process reduces the air and tissue volume of the treated lobe. Fibrosis of airways likely contributes to the atelectasis as airway narrowing/occlusion prevents airflow into distal airspaces. Both parenchymal fibrosis and atelectasis in the treated lobes correlated significantly with lobar volume reduction.

Although an initial inflammatory response in the treated regions is an expected response to treatment, a prolonged or

Table 7 Correlations between histology findings of fibrosis and atelectasis and lobar volume reduction in bronchoscopic thermal vapor ablation-treated cranial lobes

\begin{tabular}{lll}
\hline Parameter & \multicolumn{2}{l}{$\begin{array}{l}\text { Correlation with lobar } \\
\text { volume reduction }\end{array}$} \\
\cline { 2 - 3 } & r-value & P-value \\
\hline Average parenchymal fibrosis score & 0.80 & $<0.001$ \\
Average peribronchiolar fibrosis score & -0.005 & 0.984 \\
Average peribronchial fibrosis score & 0.159 & 0.556 \\
Average amount of in vivo atelectasis & 0.71 & 0.002 \\
\hline
\end{tabular}

chronic inflammatory response after treatment would not be desirable. This study shows that the inflammatory process is resolved by 3 months after treatment. At this time point, the treatment-induced effects in the lung are characterized by mature fibrosis and remodeling with associated atelectasis in the absence of inflammation. Further studies detailing the time course of pathological processes in the lung in response to BTVA using additional techniques such as immunostaining would be of benefit.

In this study, vapor ablation did not appear to affect areas of the lung other than those targeted for treatment, as the untreated middle or caudal lobes had negligible findings of fibrosis, inflammation, or tissue injury 3 months after treatment. Collateral ventilation between lobes could be one potential mechanism by which vapor could have effects in regions of the lung other than within the targeted segments. Given that the canine lung has a larger degree of collateral ventilation than does the human lung, ${ }^{17}$ this study does not support this mechanism as a possible concern.

\section{Conclusion}

In conclusion, in a canine model of emphysema, BTVA at a vapor dose of $10 \mathrm{cal} / \mathrm{g}$ results in lobar volume reduction associated with remodeling of the targeted tissue characterized by mature collagen formation without acute or chronic inflammation in the absence of major adverse events. The canine model provides objective data regarding the histopathologic mechanism of action of volume reduction and subsequent favorable clinical outcomes observed in human trials. 


\section{Acknowledgment}

This study was funded by Uptake Medical Corp.

\section{Disclosure}

The authors report no conflicts of interest in this work.

\section{References}

1. National Institutes of Health. National Heart, Lung, and Blood Institute. National Emphysema Treatment Trial (NETT): evaluation of lung volume reduction surgery for emphysema. Available from: http:// www.nhlbi.nih.gov/health/prof/lung/nett/lvrsweb.htm. Accessed December 12, 2011.

2. Global Initiative for Chronic Obstructive Lung Disease. Global strategy for the diagnosis, management, and prevention of chronic obstructive pulmonary disease. Executive summary. December 2010 Update. Available from: http://www.goldcopd.org/uploads/users/files/ GOLDReport_April112011.pdf. Accessed December 12, 2011.

3. Snell GI, Holsworth L, Borrill ZL, et al. The potential for bronchoscopic lung volume reduction using bronchial prostheses: a pilot study. Chest. 2003;124(3):1073-1080.

4. Toma TP, Hopkinson NS, Hillier J, et al. Bronchoscopic volume reduction with valve implants in patients with severe emphysema. Lancet. 2003;361(9361):931-933.

5. Hopkinson NS, Toma TP, Hansell DM, et al. Effect of bronchoscopic lung volume reduction on dynamic hyperinflation and exercise in emphysema. Am J Respir Crit Care Med. 2005;171(5):453-460.

6. Venuta F, de Giacomo T, Rendina EA, et al. Bronchoscopic lungvolume reduction with one-way valves in patients with heterogeneous emphysema. Ann Thorac Surg. 2005;79(2):411-416.

7. Wan IY, Toma TP, Geddes DM, et al. Bronchoscopic lung volume reduction for end-stage emphysema: report on the first 98 patients. Chest. 2006;129:518-526.
8. Sciurba FC, Ernst A, Herth FJF, et al. A randomized study of endobronchial valves for advanced emphysema. $N$ Eng $J$ Med. 2010;363:1233-1244.

9. Herth FJF, Gompelmann D, Stanzel F, et al. Treatment of advanced emphysema with emphysematous lung sealant $\left(\right.$ AeriSeal $\left.^{\mathbb{}}\right)$. Respiration. 2011;82(1):36-45.

10. Snell GI, Westall P, Holsworth L, Carle A, Williams TJ. A feasibility and safety study of bronchoscopic thermal vapor ablation: a novel emphysema therapy. Ann Thorac Surg. 2009;88(6):1993-1998.

11. Snell GI, Hopkins PM, Baker K, et al. The efficacy of bronchoscopic thermal ablation (BTVA) in patients with upper lobe emphysema. $\mathrm{Am}$ J Respir Crit Care Med. 2011;183:A6089.

12. Emery MJ, Eveland RL, Eveland K, Couetil LL, Hildebrandt J, Swenson ER. Lung volume reduction by bronchoscopic administration of steam. Am J Respir Crit Care Med. 2010;182:1282-1291.

13. Tuck SA, Anderson JC, Lopes-Berkas V, Beam S, Lowe N. The safety and efficacy of bronchoscopic thermal vapor ablation (BTVA) in an emphysematous canine model. Am J Respir Crit Care Med. 2011; 183:A1553.

14. Anthonisen NR. Tests of mechanical function. In: Macklem PT, Mead J, editors. Handbook of Physiology. The respiratory system, Section 3, Vol. III, Chapter 44. Bethesda, MD: American Physiological Society; 1986: 753-784.

15. Rubin LJ, Windberg P, Taylor W, Heatfield B. Pulmonary vascular structural and functional changes in papain-induced emphysema in dogs. Am Rev Resp Dis. 1987;136:704-709.

16. Martorana PA, Wüsten B, Van Even P, Göbel H, Schaper J. A six-month study of the evolution of papain-induced emphysema in the dog. Am Rev Respir Dis. 1982;126:898-903.

17. Tsai LW, Hoffman AM, Mazan MR, Ingenito EP. Bronchoscopic measurement of collateral ventilation in a sheep model of emphysema. Respiration. 2007;74:565-571. 


\section{Appendix I: detailed study methodology \\ Methods}

All procedures were reviewed and approved by the Institutional Animal Care and Use Committee of American Preclinical Sciences (Coon Rapids, MN). Unless otherwise stated, all averaged values are presented as mean \pm standard deviation.

\section{Animal model}

In order to induce emphysema-like changes in the lungs, eleven adult mongrel dogs (mean $\mathrm{wt}=20.9 \mathrm{~kg}$ ) were exposed twice to aerosolized papain ( $3 \mathrm{~mL}$ of a $4 \%$ solution) via endotracheal tube while under anesthesia. ${ }^{1}$ The two papain exposures were separated by 7 days. Just prior to the first exposure, functional residual capacity (FRC) was measured, as described in this appendix.

\section{Study design}

The papain-exposed dogs were assigned to either a treatment group (bilateral BTVA treatment; $\mathrm{n}=8$ ) or a sham group (bilateral sham treatment; $n=3$ ). The BTVA or sham procedure was performed $74-79$ days after the last papain exposure.

Prior to treatment, baseline measurements (vital signs, computed tomography [CT] scans of the chest and brain, blood work [hematology, biochemisty, coagulation, and tissue injury parameters], and FRC) were obtained. The animals then underwent bilateral BTVA or sham treatment of the upper lobes. Perioperative measurements of heart rate (HR), arterial blood pressure, and arterial oxygen saturation $\left(\mathrm{SaO}_{2}\right)$ were monitored immediately prior to and after BTVA or sham treatments. Postoperative assessments consisted of blood work (postoperative Days 1, 3, 7, 30, and 90), clinical observations (daily from Day 1 to Day 10), and chest and brain CTs at Day 30 and Day 90. At $\sim 90$ days posttreatment, the animals were euthanized, lungs removed, and visual estimates of lobar volume reduction performed, and then the lungs were processed for histological analysis.

\section{Preprocedural animal preparation and functional residual capacity measurement}

Animals were anesthetized with isoflurane in $100 \% \mathrm{O}_{2}$ and then intubated. FRC was measured by the helium dilution method. ${ }^{2}$ The FRC measurement was repeated three times and the average of the three measurements was used to determine the BTVA delivery time (see Bronchoscopic thermal vapor ablation treatment).

\section{Bronchoscopic thermal vapor ablation treatment}

The bronchoscopic thermal vapor ablation (BTVA) system comprises a vapor generator and a vapor catheter. The vapor generator is an electronically controlled pressure vessel that generates and delivers precise amounts of energy (heated vapor) through the vapor catheter and into a targeted lung segment. The vapor catheter is composed of a flexible shaft and occlusion balloon located at the distal end. The BTVA procedure was performed in intubated animals under general anesthesia. During the procedure, the vapor catheter was introduced through the bronchoscope into the airway of the lung segment selected for treatment, where an occlusion balloon was then inflated and the predetermined vapor dose $(10 \mathrm{cal} / \mathrm{g}$ of lung tissue) delivered to the targeted lung segments. A total of three segments in the cranial lobes were targeted for treatment: two segments in the right cranial lobe (the first dorsal branch in the right cranial [RB1D1] and the branch distal to the second dorsal branch [RB1-distal]) and one segment in the left cranial lobe (the branch distal to the first dorsal branch [LB1-distal]). The order of treatment was randomized.

The time of vapor delivery was based on the estimation of segmental tissue mass for each individual dog. Preliminary studies were performed using canine lung casts to determine the percentage of total measured air volume (as estimated by FRC) represented by each cranial lobe segment (RB1D $1=5 \%$ of FRC, RB1-distal $=9.3 \%$ of FRC, LB1distal $=6.4 \%$ of FRC). Segmental air volume was estimated using the appropriate percentage of FRC, then segmental mass was estimated based on density of the upper lobe region as calculated for each dog from chest CTs taken an average of 23 days (range 20-28 days) prior to treatment. A single value of lung density, in $\mathrm{g} / \mathrm{L}$, was calculated from representative slices of the left and right cranial lobes using the following relationship: density = lung attenuation in Hounsfield units $(\mathrm{HU})+1000$. The time required to deliver a dose of $10 \mathrm{cal} / \mathrm{g}$ was then calculated for each segment based on the segmental mass and the time/energy relationship of the generator used. Sham treatment consisted of placement of the catheter and balloon inflation for a time similar to that required to deliver a vapor dose of $10 \mathrm{cal} / \mathrm{g}$.

After the three vapor treatments were delivered, the animal was recovered from anesthesia and returned to its housing. 


\section{Assessment of acute cardiovascular effects}

Blood pressure, as measured by an indwelling femoral arterial catheter, and electrocardiography (ECG) were measured for 30 seconds before and after each of three successive treatments. The average heart rate and mean arterial pressure (MAP) were calculated over each 30 -second period. In addition, ECG recordings were qualitatively assessed for rhythm, and one representative ECG complex per 30-second recording was quantitatively analyzed for RR, PQ, QRS, QT, and corrected QT (QTc) intervals. The QTc intervals were calculated using the methods of Fridericia. ${ }^{3}$

An arterial blood gas sample was taken from the femoral artery just prior to treatment of the first segment (baseline) and within a minute of treatment of the third segment. The blood sample was immediately analyzed for $\mathrm{SaO}_{2}$.

\section{Clinical observations}

Clinical observations and vital signs (temperature, HR, respiratory rate, peripheral oxygen saturation by pulse oximeter) were documented daily by trained animal care personnel for at least 10 days posttreatment.

\section{Computed tomography assessment}

$\mathrm{CT}$ scans of the chest and brain were performed at pretreatment, 30 days ( \pm 7 days) posttreatment and at the time of sacrifice (90 \pm 7 days) using a mobile CT unit (Siemens Emotion 6; Munich, Germany). Lung scans were performed at both end-inspiration and end-expiration. The CT images were qualitatively evaluated by a trained radiologist. The brain scan was assessed for evidence of embolic stroke, and the chest CT scan was assessed for evidence of pneumothorax and consolidation in the treated or untreated lobes.

\section{Clinical pathology}

Blood samples were taken at pretreatment (Day 0) and at Days $1,3,7$, and 30 ( \pm 7 days) posttreatment and the day of sacrifice (90 \pm 7 days). In addition to hematology and serum biochemistry, tissue injury markers (total creatine kinase and lactate dehydrogenase) were also measured at Days $0,1,3$, and 7 .

\section{Assessment of lobar volume changes}

Approximately 90 days after treatment, the animals were euthanized by exsanguination while under general anesthesia. At necropsy, the lungs were removed and inflated with air to a pressure of $20-30 \mathrm{~cm} \mathrm{H}_{2} \mathrm{O}$. Two observers, blinded to the treatment condition, visually scored the amount of right and left cranial lobar volume reduction observed in the treated lungs in relationship to reference lungs from a normal untreated dog of similar size whose lungs had been dried while at a similar inflation pressure. The lobar volume reduction of the right cranial and left cranial lobes was expressed as percentage reduction. The average score of the two reviewers was calculated and rounded to the nearest whole number.

\section{Histopathology assessment}

The lungs were processed for histological examination by tracheal instillation of $10 \%$ buffered formalin and then submerged in formalin for a minimum of 24 hours. The lungs were then serially sliced at $\sim 5 \mathrm{~mm}$ intervals. For the treated right and left cranial lobes, every other slice was sampled in its entirety and placed in cassettes for histologic processing. For the untreated lobes (left and right middle, left and right caudal), only the largest slice was sampled and placed in cassettes for processing. Tissue cassettes were embedded in paraffin, sectioned at $\sim 5 \mu \mathrm{m}$, and stained with hematoxylineosin and Masson's trichrome.

The tissue slides were assessed for three categories of findings: fibrosis, inflammation, and tissue damage. Fibrosis was further defined according to the maturity of the response (immature and mature granulation tissue versus mature fibrosis) as well as the distribution (parenchymal, peribronchial, peribronchiolar, and alveolar septal). Inflammation was further defined as acute (numerous viable or degranulated neutrophils within alveolar air spaces or septa with small amounts of necrotic or pyknotic debris), chronic with ongoing tissue damage and repair (influx of mononuclear cells, including macrophages, lymphocytes, and plasma cells, and small numbers of neutrophils), or chronic without evidence of ongoing tissue damage and repair (macrophages, mixed inflammation of predominantly macrophages with rare to few neutrophils, or hemosiderophages in the airspaces/alveolar septa). Tissue damage was assessed according to evidence of vascular leakage, edema fluid, or fibrin accumulation. The proportion of slides with a given finding, expressed as a percentage of the total number of slides examined, was reported for each lobe.

\section{Correlation between fibrosis, alveolar collapse, and lobar volume reduction}

Each finding of fibrosis (peribronchial, peribronchiolar, and parenchymal) was assigned a qualitative score based on a combination of the amount and density of fibrosis present 
$(1=$ minimal, $2=$ mild, $3=$ moderate, and $4=$ severe $)$. For each right and left cranial lobe from the BTVA-treated dogs, the average severity score was calculated across all slides from the lobe. The average severity score for peribronchial, peribronchiolar, and parenchymal fibrosis for each animal was correlated with the amount of lobar volume reduction observed at the time of necropsy. For this analysis, right and left lobes were combined.

Each slide was also assessed for in vivo atelectasis, defined as incomplete expansion of alveolar air space, which most likely occurred in vivo (as opposed to during tissue processing). The atelectasis was scored according to the approximated percentage of the lung tissue involved on each slide, in increments of $5 \%$. If present but in a very low amount (ie, $<5 \%$ ), a score of $2.5 \%$ was recorded. The average amount of in vivo atelectasis for each animal was correlated with the amount of lobar volume reduction observed at the time of necropsy. For this analysis, right and left lobes were combined.

\section{Statistical analysis}

Paired $t$-tests were used to compare pretreatment to posttreatment values for ECG intervals (PR, QRS, RR, QT, QTc),
$\mathrm{SaO}_{2}, \mathrm{HR}$, and MAP. An unpaired $t$-test was used to compare lobar volume reduction between BTVA-treated and shamtreated groups. Pearson product moment correlations were used to detect the presence of an association between the amount of lobar lung volume reduction and the histological findings of fibrosis and in vivo atelectasis. A significance value of $P<0.05$ was used.

\section{References}

1. Rosenthal FS. Aerosol probes of lung injury in a 28-wk longitudinal study of mild experimental emphysema in dogs. J Appl Physiol. 1999;86:725-731.

2. Anthonisen NR. Tests of mechanical function. In: Macklem PT, Mead J, editors. Handbook of physiology. The respiratory system, Section 3, Vol. III, Chapter 44. Bethesda, MD: American Physiological Society; 1986:753-784.

3. Fridericia LS. The duration of systole in an electrocardiogram in normal humans and patients with heart disease [Die systolendauer im elektrokarfiogramm bei normalen menchan und bei herzdranken]. Acta Med Scand. 1920;53:469-486. [German.]

\section{Publish your work in this journal}

The International Journal of COPD is an international, peer-reviewed journal of therapeutics and pharmacology focusing on concise rapid reporting of clinical studies and reviews in COPD. Special focus is given to the pathophysiological processes underlying the disease, intervention programs, patient focused education, and self management protocols.

\section{Dovepress}

This journal is indexed on PubMed Central, MedLine and CAS. The manuscript management system is completely online and includes a very quick and fair peer-review system, which is all easy to use. Visit http://www.dovepress.com/testimonials.php to read real quotes from published authors. 